\title{
La profesión dental en España. Análisis de la situación actual a partir de las ofertas formativas de educación continuada. Estudio piloto

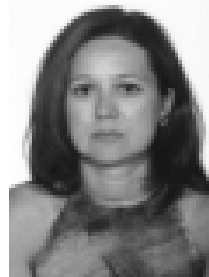 \\ Martín-Pérez, María Jesús
}

\section{Dental profession in Spain. Analysis of the current situation considering the offers \\ for continuing education studies. An initial approach}

\author{
Martín- Pérez, Ma lesús* \\ Mallo- Pérez, Luciano**
}

*Médico Estomatólogo.

**Doctor en Medicina y Cirugía. Estomatólogo.

\begin{abstract}
Resumen: Objetivo: interpretar la orientación actual de la profesión dental en España, a partir de los datos aportados por la oferta de formación postgraduada.

Material y método: se han clasificado las 529 ofertas formativas que aparecieron en 3 números consecutivos de las revistas Gaceta Dental (3 meses) y Maxillaris (3 meses) entre septiembre de 2002 y febrero de 2003, según su tema, duración y organizador. También se analizaron los libros de formación continuada editados por el Consejo General de Colegios de Odontólogos y Estomatólogos de España, correspondientes a los años 2002 y 2003 (en total 203 entradas). Resultados: el $27,5 \%$ de los cursos ofertados eran de contenido implantológico. Le siguen en frecuencia aquellos relativos a ortodoncia $(17,6 \%$ y periodoncia $(6,1 \%)$. La gran mayoría de la oferta $(68,5 \%)$ estaba organizada por iniciativa privada. El $43,3 \%$ de las actividades que se ofertaron eran de corta duración, el 27,8\%de duración intermedida y el 27,8\%tenían una duración prevista mayor de 60 horas lectivas. De los cursos propuestos por los Colegios Oficiales de Odontólogos y Estomatólogos y por su Consejo General, el 70,5\% tienen una orientación marcadamente técnica o tecnológica.
\end{abstract}

Palabras clave: Profesión dental, Tendencia actual, Cursos de formación.

Abstract: Objetive: to understand the current trends in the dental profession in Spain, from the analysis of the offers for postgraduate studies.

Material and method: The analysis was performed on a total of 529 offers appearing on 3 consecutive issues of the journals Gaceta Dental ( 3 months) and Maxillaris ( 3 months), between September 2002 and February 2003. Their subject, duration and organizer were considered. In addition, the annual reports of the General Council of the Professional Associations of Odontology and Stomatology of Spain corresponding to the years 2002 and 2003 (203 entries) were analyzed.

Results: Courses on implantology were the most frequently offered (27.5\%), followed by those on orthodontics (17.6\%) and periodontics (6.1\%). Most of the offers $(68.5 \%$ were organized by private initiatives. $43.3 \%$ were short courses, $27.8 \%$ were of intermediate duration and $27.8 \%$ of the courses offered more than 60 teachy hours. From the courses offered by the Official Professional Associations of Odontology and Stomatology, 70.5\% are technically or technologically oriented.

Key words: Dental profession, Current trends, Postgraduate courses.

\begin{tabular}{ccc}
\hline Fecha recepción & Fecha última revisión & Fecha aceptación \\
$03-09-2003$ & $06-10-2003$ & $07-10-2003$
\end{tabular}

BIBபD [1138-123X (2003)8:6; noviembre-diciembre 593-724]

Luciano Mallo Pérez.

Av. España, 33, 1ำ.

24400 Ponferrada (León)
Martín-Pérez MJ , Mallo-Pérez L La profesión dental en España. Análisis de la situación actual a partir de las ofertas formativas de postgrado. Estudio piloto. RCOE 2003;8(6):611-619. 


\section{Introducción}

Querámoslo o no, la civilización occidental actual se puede (o se intenta) reducir a cifras. Cualquier persona, colectivo, entidad, empresa, o administración, vive esclavizado por una miríada de balances, informes, objetivos, previsiones, etc. Todo ello es posiblemente necesario, incluso imprescindible, pero, al mismo tiempo, también resulta en ocasiones desproporcionado y esclavizante. De cualquier modo, la realidad presente, y más aún la futura, está planteada de esta manera y a ella no nos queda otro remedio que adaptarnos.

La ciencia y la profesión médica, y también la bucal y dental, tampoco se pueden escapar de esta maraña de cifras, ya sea en forma de índices biológicos (aquellos que traducen a números situaciones o estados físiológicos o patológicos), de la manipulación racional matemática de los mismos (el complejo mundo de la Bioestadística y la Epidemiología), de la interpretación de éstos (informes, previsiones, recomendaciones y política sanitaria) o, al fin, de los números alrededor de los cuales gira el complejo cultural actual (los dineros y su gestión).

Debiera ser una ilusión para todos los practicantes, en este caso los dentistas, conocer su pasado; pero lo que ciertamente constituye una obsesión para todos ellos, ya sea a título individual o colectivo (asociaciones, colegios, sociedades), es conocer el presente y más aún el futuro de la profesión. Con este fin, existen numerosos y periódicos informes de expertos que analizan transversalmente la situación para establecer previsiones

\begin{tabular}{|c|c|}
\hline \multicolumn{2}{|c|}{ Tabla l: Clasificación de la oferta de cursos según su contenido* } \\
\hline Periodoncia & Salud Pública- Prevención \\
\hline Implantes & Gestión- Marketing \\
\hline Ortodoncia & Radiología dental \\
\hline Prótesis & Ergonomía \\
\hline Endodoncia & Odontopediatría \\
\hline Cirugía oral & Materiales odontológicos \\
\hline Operatoria dental- Conservadora & Urgencias \\
\hline Tecnología & Odontogeriatría \\
\hline Estética dental & Odontología del deporte \\
\hline Medicina oral & Pacientes especiales \\
\hline ATM & Multidisciplinario \\
\hline Oclusión & \\
\hline \multicolumn{2}{|c|}{$\begin{array}{l}\text { * En muchos casos fue muy difícil optar por una categoría determinada, ya fuese } \\
\text { por que la oferta no era clara o explícita o porque muchas de las categorías com- } \\
\text { parten espacios comunes. }\end{array}$} \\
\hline
\end{tabular}

futuras. Así, disponemos de varios informes sobre la situación epidemiológica de la población ${ }^{1-5^{*}-7}$ de la cantidad y distribución de la mano de obra denta ${ }^{8,9}$ incluso de la actividad profesional $1^{10,11^{* * 112}}$. Sin embargo, que nosotros conozcamos, no existe ningún trabajo en España que tome como criterio de medida la oferta de actividad docente postgraduada.

Es el objetivo del presente artículo tratar de conocer la orientación de la profesión dental en España, utilizando un sistema de medición poco habitual, con el ánimo de aportar un punto de vista, ya no sólo diferente, sino más bien complementario en esta cuestión.

\section{Material y método}

Hemos utilizado como fuente los números de la revista Gaceta Dental correspondientes a los meses de septiembre y noviembre de 2002 y enero de 2003 y los de la revista Maxillaris de los meses de octubre y diciembre de
2002 y febrero de 2003, cubriendo así seis meses consecutivos.

Para poder establecer una comparativa retrospectiva, hemos utilizado los números correspondientes a los mismos meses, pero de 10 años antes (1992-93), todos de la revista Gaceta Dental , ya que en aquellos momentos la revista Maxillaris todavía no se publicaba.

También utilizamos los libros oficiales de cursos organizados o patrocinados por el Consejo General de Colegios de Odontólogos y Estomatólogos de España y los distintos Colegios Regionales y Provinciales, de los años 2002 y 2003.

En el caso de las publicaciones Gaceta Dental y Maxillaris, se han clasificado todos los cursos ofertados en cualquiera de sus secciones, de acuerdo con 23 categorías, según su contenido (tabla 1). Para clasificar los organizadores de los cursos se utilizaron 7 categorías excluyentes (tabla 2), y la duración se estimó según 3 posibilidades también incompatibles entre 


\begin{tabular}{|c|}
\hline Tabla 2: Clasifícación de los \\
CurSos según el organizador \\
\hline Universidad \\
\hline Colegio Profesional \\
\hline Sociedad Científica \\
\hline Clínica en clínica (A) \\
\hline Clínica en hotel (B) \\
\hline Asociación circunstancial \\
en clínica (C) \\
\hline Asociación circunstancial \\
en hotel (D) \\
\hline (A) Clínica unipersonal o pluriperso- \\
nal, con plantilla profesional estable y \\
reconocida y de experiencia o presti- \\
gio (publicaciones, cursos, conferen- \\
cias, etc.) en sus instalaciones. \\
(B) Lo mismo, pero fuera de sus ins- \\
talaciones y en lugares propios de \\
conferencias (salas de hotel, audito- \\
rios de empresas, etc.) \\
(C) Entidad, empresa o profesional o \\
grupo de ellos que se reúnen para \\
una actividad determinada en una clí- \\
nica dental, habitualmente diferente a \\
la que trabajan. Se incluyen también \\
aquellas clínicas cuyo personal varía \\
con frecuencia, siendo el único ele- \\
mento constante el empleador o, nor- \\
malmente, la empresa gestora de la \\
misma. \\
(D) Lo mismo pero en las condiciones \\
que se exponen en el apartado (B). \\
\hline
\end{tabular}

sí. Igualmente se incluyeron tres posibilidades no siempre necesarias (conferencia magistral, congreso periódico y título oficial), compatibles con cualquier tipo de organizador y duración. Se ha tenido especial cuidado en no valorar una misma oferta si aparecía dos o más veces en la misma revista; sin embargo esto no se tuvo en cuenta entre números o revistas diferentes, a pesar de que muchos cursos se ofertaban varios meses seguidos $y$ casi siempre en las dos publicaciones al mismo tiempo.

La valoración de la temática se hizo según el programa del curso, si éste aparecía en la oferta; en caso contrario, nos guiamos por el nombre del mismo, casi siempre revelador. Lo mismo ocurrió para el caso de la duración, aunque ésta casi siempre aparecía reflejada. Consideramos como unidad de medida de duración el "módulo" al que definimos como una actividad formativa de 1 día o 2 días consecutivos (habitualmente un fin de semana $y$ menos de 12 horas lectivas).

Para los cursos ofertados a través de los Colegios Oficiales de Odontólogos y Estomatólogos, se establecieron tres posibilidades excluyentes entre sí: de contenido «écnico-tecnológico», «no técnico-tecnológico» o bien «mixto». Para su valoración se utilizó el contenido del programa que de cada curso aparece siempre en la oferta correspondiente. Se consideró «técnico-tecnológico» a aquella actividad formativa que, prioritaria o exclusivamente, tratase de temas relacionados con una o varias técnicas clínicas manipulativas, materiales o aparataje. Se consideró «no técnico-tecnológico» a aquellos cursos en que predominantemente se tocasen temas clínicos, etiológicos, fisiopatológicos, diagnósticos o de tratamiento, en los que, aunque se utilizase tecnología, lo importante fuese lo que se hace 0 consigue con la misma y no como se maneja o manipula. Finalmente, cuando se mezclaban aspectos técnicos y no técnicos, y no pudimos o supimos clasificar claramente en alguna de las categorías anteriores, se optó por la opción de «mixto».

Para la interpretación de los datos no se utilizó ningún análisis estadístico convencional, sino una interpretación cuantitativa y cualitativa de los mismos.

\section{Resultados y discusión}

\section{A. Referido al material y método.}

Tanto la Medicina, en todas sus ramas, como la Odontología han sufrido una espectacular evolución en las últimas décadas, ya no sólo en cuanto a su vertiente científica, sino también referido al perfil profesional y laboral. La mayoría de los practicantes muestran curiosidad e inquietud por dichos cambios y la medida en que éstos puedan influir en su actividad cotidiana.

En Epidemiología siempre se han recomendado los estudios objetivos y directos para conocer el estado de la cuestión; sin embargo, a veces éstos no son posibles o factibles, son muy complicados, no son viables, fiables 0 realistas. Muchos estudios en Demografía y Sociología emplean indicadores de medida indirecta, que, aunque no miden directamente la variable que se pretende estudiar, lo hacen estudiando sus consecuencias y repercusiones y tantas veces obtienen resultados a los que se les otorga un grado máximo de confiabilidad. Así, por ejemplo, para conocer el grado de seguimiento de una huelga general se valora el consumo energético $o$ para saber los movimientos de población en las zonas turísticas se valora el consumo de agua y las toneladas de basura que se generan y ello se considera mucho más fiable que los datos aportados por la patronal y los sindicatos o los de ocupación hotelera, respectivamente ${ }^{13}$.

De igual modo, hemos empleado 


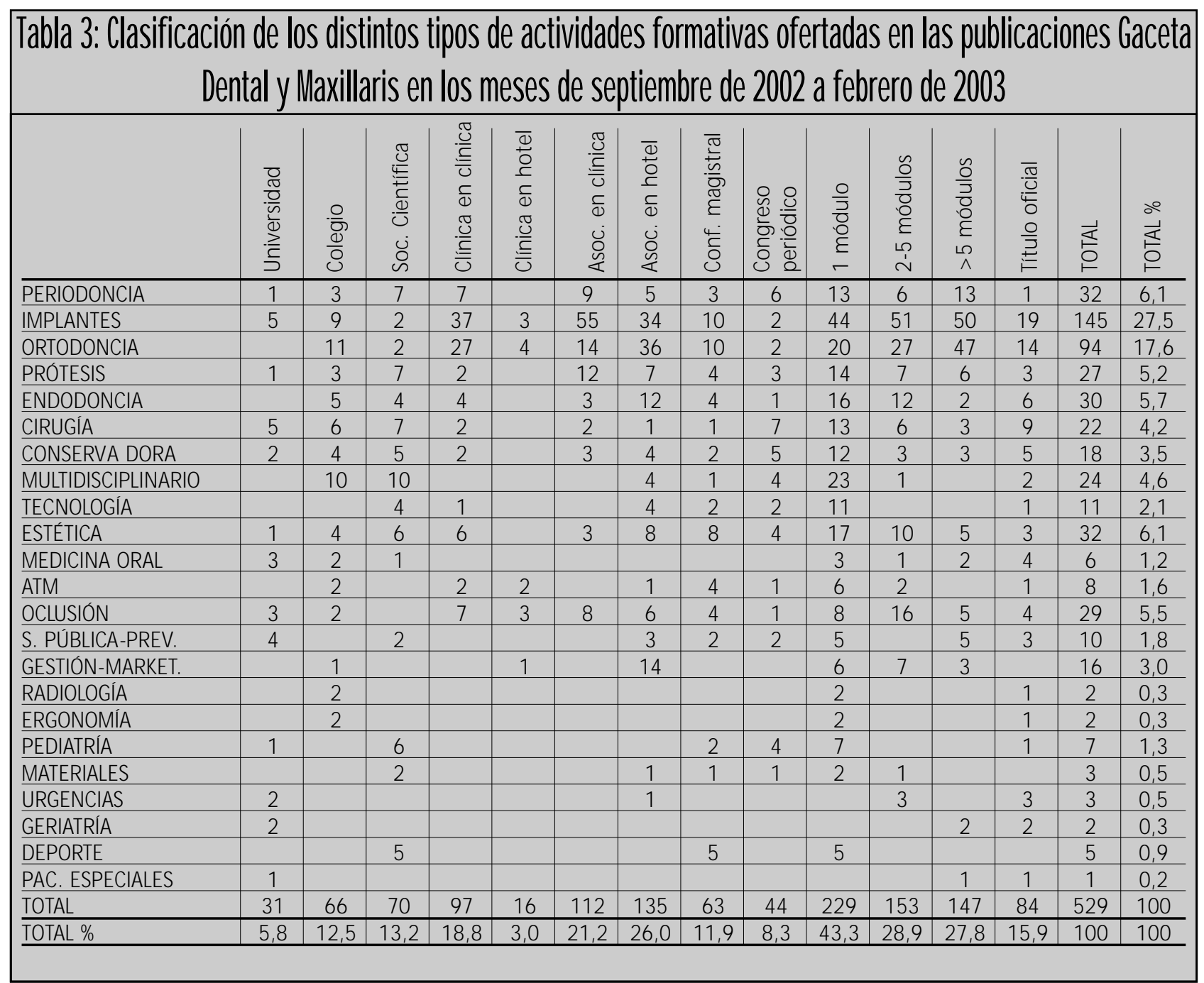

como elemento de medida la oferta de cursos de formación odontológica o estomatológica. Es una constante en Biología, y también en Economía, que la relación entre la oferta y la demanda es directamente proporcional, de manera que ambas tienden al equilibrio. Por ese motivo utilizamos este elemento de medición, poco convencional, poco o nada frecuente, pero consideramos que bastante operativo y demostrativo.

Utilizamos las revistas Gaceta Den- tal y Maxillaris como fuente, por su implantación máxima en el sector dental (colegiados y no colegiados, convalidados y no convalidados, protésicos, higienistas, etc.), porque prácticamente todos los cursos de postgrado se ofertan en las mismas, por su carácter eminentemente divulgativo, por su periodicidad mensual, por su gran puntualidad y por ser de distribución gratuita. Revisamos 6 números consecutivos (medio año), para hacernos una idea realista de la oferta general de cursos - en total 529, estimamos que muy representativa- y alternamos los números de una y otra publicación para descartar posibles sesgos, francamente poco probables ya que la oferta de actividades formativas en una y otra revista en el mismo mes es casi superponible.

Descartamos para este fin como fuente la revista oficial del Consejo General de Colegios de Odontólogos y Estomatólogos de España (RCOE), por ser ésta una revista esencialmente 


\begin{tabular}{|c|c|c|}
\hline $\begin{array}{l}\text { Tabla 4: Clasificación } \\
\text { frecuencia en los año }\end{array}$ & $\begin{array}{l}\text { la oferta de cursos f } \\
002-3 \text { y 1992-3, ofer } \\
\text { ta Dental y Maxillaris }\end{array}$ & $\begin{array}{l}\text { ormativos, según su } \\
\text { tados en las revistas }\end{array}$ \\
\hline ORDEN DE FRECUENCIA & 2002-2003 & 1992-1993 \\
\hline Primero & Implantes $(27,5 \%)$ & Implantes $(31,7 \%)$ \\
\hline Segundo & Ortodoncia $(17,6 \%)$ & Ortodoncia $(17,5 \%)$ \\
\hline Tercero & $\begin{array}{c}\text { Periodoncia }(6,1 \%) \\
\text { Estética }(6,1)\end{array}$ & Periodoncia $(6,9 \%)$ \\
\hline Cuarto & & Prótesis $(5,3 \%)$ \\
\hline Quinto & Endodoncia (5,7\%) & $\begin{array}{c}\text { Conservadora }(5,0 \%) \\
\text { Multidisciplinario }(5,0 \%)\end{array}$ \\
\hline Sexto & Oclusión $(5,5 \%)$ & \\
\hline Séptimo & Prótesis $(5,1 \%)$ & $\begin{array}{c}\text { Endodoncia }(4,3 \%) \\
\text { Cirugía }(4,3 \%)\end{array}$ \\
\hline Octavo & Multidisciplinario $(4,6 \%)$ & \\
\hline Noveno & Cirugía $(4,2 \%)$ & Medicina Oral $(3,8 \%)$ \\
\hline Décimo & Conservadora $(3,5 \%)$ & $\begin{array}{c}\text { Salud Pública- } \\
\text { Prevención }(2,4 \%)\end{array}$ \\
\hline
\end{tabular}

científica, con un carácter divulgativo secundario y en la que habitualmente no se publicitan cursos que no estén organizados por los Colegios Oficiales, el Consejo General o las Sociedades Científicas a él adscritas, y que como hemos podido comprobar suponen menos de la tercera parte del total de cursos que se organizan.

\section{B. Referido a los resultados.}

Hemos registrado un total de 529 propuestas formativas publicitadas en las revistas Gaceta Dental y Maxillaris, en seis meses consecutivos entre septiembre de 2002 y febrero de 2003. La descripción detallada y clasificada de éstos se puede consultar en la tabla 3.

L primero que llama la atención es que la oferta de cursos de implantes supera el $25 \%$ del total y que hay 3 disciplinas (implantes, ortodoncia y periodoncia) que conjuntamente suponen el $51,2 \%$ de las propuestas.
Solamente las actividades postgraduadas referidas a implantes y ortodoncia tienen frecuencia superior al $17 \%$ estando el resto de la oferta muy atomizada. En la tabla 4 se puede ver la clasificación ordenada de las disciplinas según el porcentaje de cursos formativos que aparecen ofertados en las revistas antes señaladas.

La implantología actual tiene apenas 30 años, aunque su optimización, popularización y universalización es una conquista de la última docena. Como tal, al menos en la actualidad, la implantología no existe como una disciplina independiente, como puede ser la Ortodoncia por ejemplo, y para sí se la intentan atraer (todos con razones fundamentadas) desde cirujanos -orales, maxilofaciales- hasta odontólogos y estomatólogos generales, pasando por periodoncistas, protesistas, especialistas en estética, por no citar a otras especialidades médicas como ORL, traumatología y cirugía plástica. Tal atracción multipolar de-muestra una doble característica: por un lado su enorme versatilidad y posibilidades terapéuticas y por otro, tan valorable como el anterior sino más, las implicaciones económicas de los tratamientos implantológicos, hoy por hoy muy costosos y no asumidos por el sistema público de salud. Hasta tal punto, y por los motivos que fuere, es interesante la implantología que incluso una sociedad científica odontológica, probablemente la más influencial y con más miembros, se ha negado a sí misma para incluir entre sus objetivos propios los implantológicos. Curiosamente, hace 10 años el porcentaje de cursos relacionados con la implantología era mayor, aunque en números absolutos representa la tercera parte (tabla 5).

La segunda disciplina en el orden de frecuencia de la oferta fue la ortodoncia y el tercer lugar lo ocupa la periodoncia y así también lo era una década antes (tabla 4).

Estas tres disciplinas (implantología, ortodoncia y periodoncia) en conjunto suman más de la mitad de la oferta formativa de postgrado y esto puede ser un interesante tema de comentario, fundamentado en tres características, como mínimo peculiares: a) la formación de pregrado en estas tres materias, sobre todo en su aspecto práctico, es claramente deficitaria; casi cualquier estomatólogo u odontólogo sale de las escuelas o facultades en condiciones de efectuar obturaciones, endodoncias o exodoncias, pero no para afrontar tratamientos periodontales, implantológicos u ortodóncicos. Desde el pregrado se ha impuesto el axioma de que son tratamientos altamente complejos y 


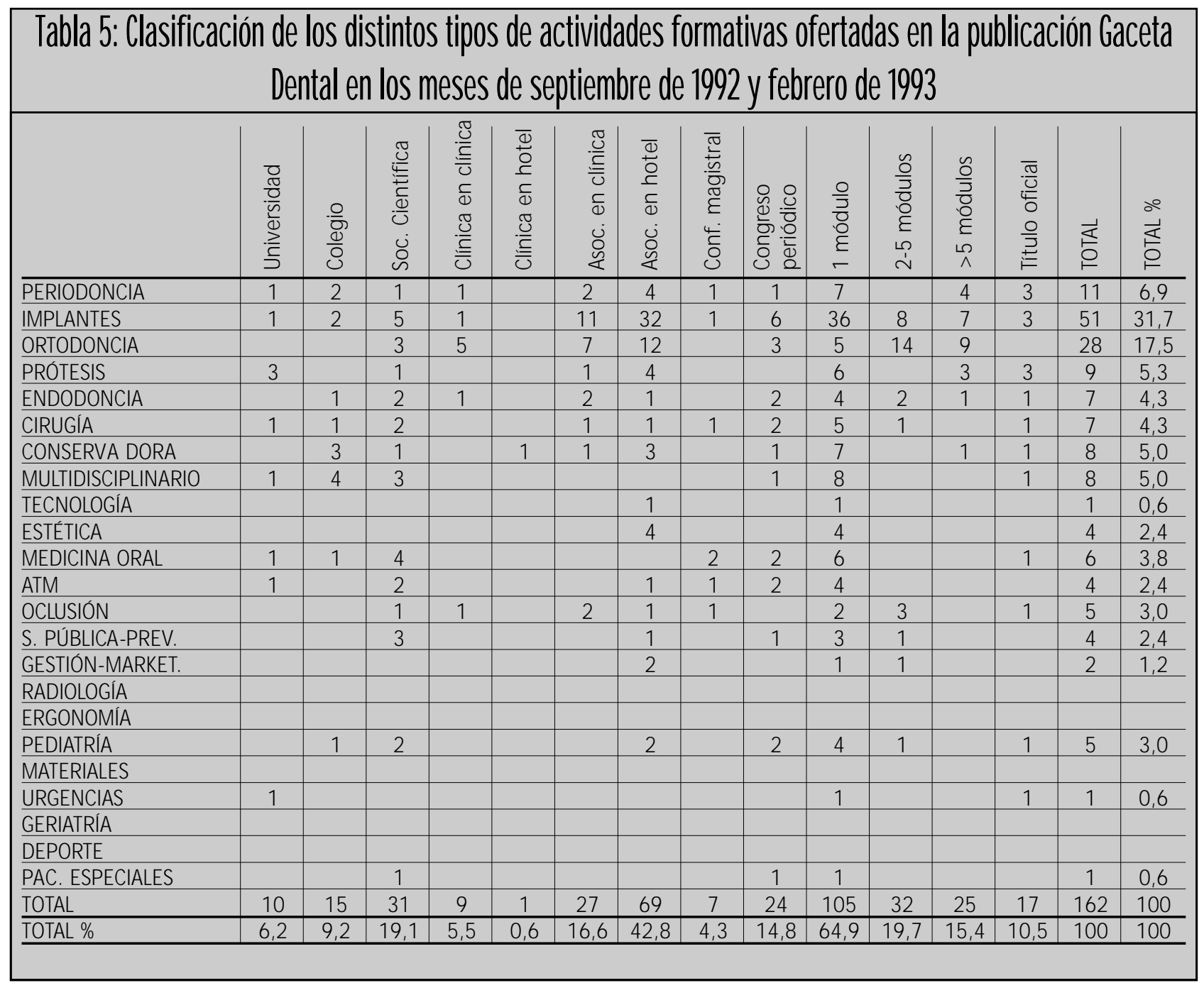

especializados y que deben afrontarse desde una situación de postgrado. b) Los estudios relativos al tiempo de consulta de los odontólogos (y estomatólogos) como conjunto, revelan que el tiempo dedicado a estas tres disciplinas es menos del $20 \%$ del total $^{11^{* *}, 14}$. No deja de ser curioso, casi paradójico, que la mayoría de la oferta de cursos se concentra en lo que es minoritario en el tiempo de consulta. c) Hoy por hoy, estas tres disciplinas engloban a los especialistas, subespe- cialistas, superespecialistas, o como se les deba o quiera llamar, mejor considerados profesional y socialmente. Además, son los que son capaces de generar mayores ingresos, que en el mundo actual es un importantísimo valor añadido, para tantos el que más.

«En la actualidad, prácticamente no se programan cursos sobre prótesis completa ni parcial extraible (chapeau Dr. Salsench, por fin alguien se da cuenta que «sacar» no es lo mismo que «remover») y de hecho los princi- pios de dimensión vertical, situación del plano oclusal y relación centrada se aprenden al llevar a la práctica este tipo de restauraciones». «Temas tan presentes como la prevención dental, el principio de sellado en endodoncia, de la restauración con materiales en la caries dental con efecto duradero y de larga vida ¡cuán olvidados están!» Ambos párrafos están tomados literalmente de un magnífico y realista reciente editorial ${ }^{15 * *}$, que se refrenda plenamente con los resultados del 


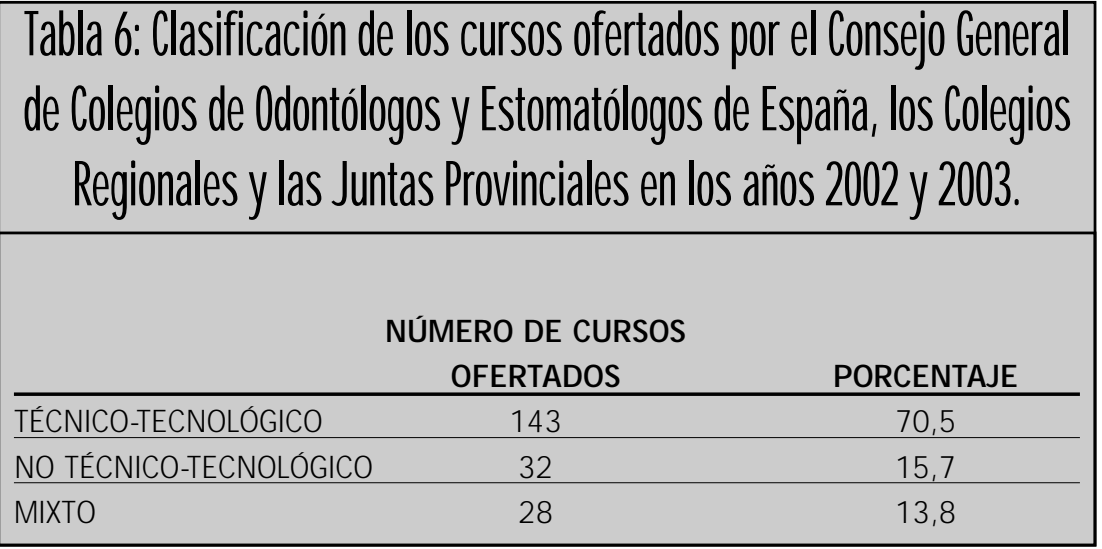

presente estudio y que no hacen sino reflejar que, a pesar del enorme progreso y evolución de la Odontología, los fundamentos científicos y técnicos hace mucho tiempo que están descritos y contrastados y que, a pesar de ser la base de la mayoría del tiempo de trabajo de los dentistas como colectivo, cada vez se arrinconan más por un progreso no siempre basado en la evidencia científica sóli$\mathrm{da}^{9,11^{* *}, 12,15^{* *} \text {. }}$.

Diez años antes la oferta de cursos era mucho más pequeña; clasificamos 162 propuestas, lo que constituye menos de la tercera parte de lo visto en 2002-03. También comprobamos que entonces la disciplina más comunmente ofertada era la implantología, casi con un tercio del total y que la oferta de formación postgraduada de implantes, ortodoncia y periodoncia suponía casi el $60 \%$ del total (tabla 5). Al comparar las propuestas de actividades formativas con 10 años de intervalo, vimos que son bastante constantes los porcentajes que corresponden a cada disciplina; probablemente, lo más destacable sea el gran aumento en la oferta de cursos de estética dental (tabla 4).
En cuanto a los organizadores de las actividades formativas, pudimos observar que casi la mitad de la oferta (concretamente el 47,2\%) correspondía a lo que hemos denominado «asociación temporal». En esta categoría, bastante heterogénea, quisimos incluir a todos aquellos organizadores que reúnen a uno o varios profesionales para ese curso concreto 0 para una serie de ellos, y lo hacen normalmente con una finalidad de lucro directo (derechos de inscripción) o indirecto (por los materiales o productos que se emplean y recomiendan en el mismo); son frecuentes los denominados «grupo de trabajo de» $y$ «asociación para el estudio de» y también las empresas de distribución y venta de material (principalmente de implantes y ortodoncia) que organizan actividades formativas con un perfil acorde con su catálogo. Con este tipo de organizadores prevalecen las actividades que se realizarían en locales de reunión, como auditorios, salas de hotel, de convenciones o de empresas, etc., sobre las que se organizan en una clínica ( $26 \%$ y $21,2 \%$ ); ello habla de que, aunque se instruya sobre la vertientes técnica de los tra- tamientos, predominan las enseñanzas de presentación sobre las manipulativas directas. Todo lo contrario ocurre cuando el organizador era una clínica estable, solvente y habitualmente de referencia, que en la mayoría de los casos (18,3\% frente a $3 \%$, organizan cursos en sus propias instalaciones y la orientación de los mismos, ya no sólo es práctica, sino principalmente manipulativa (tabla 3).

Prácticamente el mismo patrón se observó al analizar los organizadores de propuestas educativas diez años atrás. No obstante, entonces eran incluso más frecuentes los cursos organizados por lo que denominamos sasociación temporal en hotel» (tabla 5).

Las entidades que tienen capacidad institucional en la formación de postgrado ofertan menos de un tercio del total de propuestas (31,5\%); esta observación apenas varió en 10 años (tablas 3 y 5 ).

Hemos visto que el $11,9 \%$ de los cursos encajarían en lo que denominamos «conferencias magistrales», es decir aquellas impartidas por un ponente de reconocido prestigio nacional, y casi siempre internacional, que expone sus conceptos, técnicas 0 pone al día en alguna rama del conocimiento o de la técnica. Está claro que es muy difícil discernir entre lo que es un «conferenciante de reconocido prestigio» del resto y, además, esto no garantiza que la calidad del curso sea siempre superior a la de aquellos otros impartidos por otras personas que no entren en esta categoría curricular.

También es de destacar que menos de la décima parte de las propuestas correspondan a los congresos periódicos, normalmente anuales, de las 
Sociedades Científicas, siendo hace 10 años esa proporción el doble. Bien es cierto que no todos, aunque sí casi todos, los congresos de las Sociedades Científicas se publicitan en las revistas analizadas; de cualquier manera, esto nos indica que cada vez es mayor el protagonismo de la iniciativa privada a la hora de organizar cursos y otras actividades de postgrado ${ }^{16^{*}}$.

Otro aspecto interesante lo constituye la duración de los cursos. La mayor parte $(43,3 \%$ son cursos de corta duración, de menos de 12 horas lectivas, habitualmente desarrollados a lo largo de un fin de semana. Sin embargo, son también muchas las propuestas de cursos con 2 a 5 módulos (28,9\%) y los de mayor duración, de 6 o más módulos $(27,8 \%$ ). Pudimos comprobar que casi todos los cursos de larga duración se daban en las disciplinas de implantes y ortodoncia, que son, al mismo tiempo, las que tienen mayor oferta docente. Si comparamos estos datos con lo visto diez años antes, comprobamos que se ha producido un notable incremento de los cursos y actividades formativas de mayor duración y un descenso proporcional de las más cortas (tablas 3 y 5).

Solamente en el $15,9 \%$ de los cursos se otorgan títulos con validez oficial, entendiendo por ello aquellos expedidos por entidades con capacidad docente y formativa y aquellos a los que se concede la acreditación docente por el Ministerio correspon- diente (Educación o Sanidad). El hecho de otorgar un título oficial no garantiza la calidad del curso, ni que sea mejor que otro sin esta calificación, pero, al menos en teoría, significa un mejor control de calidad. El número y porcentaje de actividades de formación continuada que otorgan titulación oficial aumentó en la última década (tablas 3 y 5).

En los últimos años el Consejo General de Colegios de Odontólogos y Estomatólogos de España ha confeccionado, editado y distribuido un libro-guía con los cursos, aulas clínicas, conferencias y otras actividades de formación continuada que él mismo organiza o patrocina y con los que se preparan en los distintos Colegios Regionales y J untas Provinciales. Tienen estos documentos la gran ventaja de poder disponer en un solo volumen de la práctica totalidad de la oferta formativa de las instituciones colegiales, ordenadas por temas, fe-chas y localidades. Además, de cada actividad se presenta un resumen del contenido y se ofrece un curriculum de los dictantes. Esto nos permite conocer con gran exactitud la materia que se va a tratar y el enfoque que se tiene pensado dar a la misma. Hemos intentado establecer una clasificación casi dicotómica, entre contenidos esencialmente técnicos o tecnológicos y no técnico-tecnológicos, introduciendo un tercer apartado, que hemos denominado «mixto», para englobar aquellos que no pudimos 0 supimos asignar a una de las categorías anteriores. Es evidente que cualquier actividad formativa participa de aspectos técnicos y tecnológicos y de otros más básicos y conceptuales, con un rango que va desde lo puramente epistemológico a lo exclusivamente manipulativo; aún así resulta en la mayoría de los casos sencillo, basándonos en la información aportada por el programa, encuadrar la propuesta en una de las categorías precitadas (tabla 6).

Según lo visto en los catálogos de 2002 y 2003, comprobamos que la gran mayoría $(70,5 \%$ de los cursos tienen una orientación marcadamente técnica o tecnológica, mientras que en la minoría $(15,7 \%)$ lo era esencialmente conceptual-teórica. Estos datos no se pueden considerar, por sí mismos, buenos o malos, pero sí que pueden ser indicativos de la tendencia que tiene en la actualidad la profesión dental| ${ }^{12,14}$, máxime cuando la fuente de la que emanan los datos es la propia organización colegial dental y el número de propuestas es francamente significativo (203).

De todos estos datos podemos inferir que de seguir esta línea de oferta formativa, la profesión dental camina hacia convertirse en una disciplina esencialmente técnica y tecnológica, basada en unos fundamentos biológicos determinados, cuyo ritmo de progreso es mucho menor que el que está ocurriendo con la vertiente técnica y/o tecnológica. 


\section{Bibliografía recomendada}

Para profundizar en la lectura de este tema, el/los autor/es considera/an interesantes los artículos que aparecen señalados del siguiente modo: *de interés $* *$ de especial interés.

1. Gimeno de Sande A, Sánchez B, Viñez J, Gómez F, Mariño F. Estudio epidemiológico de la caries dental y patología bucal en España. Rev San Hig Pub 1971;45:361-433.

2. Cuenca E. La encuesta de la OMS sobre la salud buco-dental en España. Una aproximación personal. Arch Odontoestomatol 1986;2:15-22.

3. Sicilia A, Cobo J, Noguerol B et al. Prevalencia de caries en los niños y jóvenes escolares españoles de siete, doce y quince a diecinueve años. Av Odontoestomatol 1990;6:323-30.

4. Noguerol B, Llodra JC, Sicilia A, Follana M. La salud bucodental en España. 1994. Antecedentes y perspectivas de futuro. Madrid: Avances, 1995.

5*. Llodra-Calvo JC, Bravo-Pérez M, Cortés-Martinicorena, FJ. Encuesta de Salud Oral en España (2000). RCOE 2002;7 (número especial). Monográfico referido a la situación epidemiológica bucodental de la población española. Tiene el valor de ser el más reciente y el más completo de todos.

6. Cuenca E, Alvarez M. Evolución de la salud bucodental en España en los últimos 20 años. Arch Odontoest Prev y Com 1991;3:33-9.
7. Vargas F, Robledo T, Espiga I, Hernández R. Evaluación de la salud dental en España. Arch Odontoest Prev y Com 1994;10:175-85.

8. Follana M, Noguerol B, Llodra JC, Sicilia A. Estudio continuado de las necesidades de atención española. I. Demografía de la profesión dental española. 1993. Rev Actual Odontoest Esp 1994;Monográfico.

9. American Dental Association, Survey Center. 1999 survey of dental services rendered. Chicago: American Dental Association, 2002.

10. Bravo M. Age-period-cohort analysis of dentist use in Spain from 1987 to 1997. An analysis from the Spanish National Health Surveys. Eur J Oral Sci 2001;109:149-54.

$11^{* *}$. Seldin LW (por el Oversight Committee, American Dental Association of Dentist Report). El futuro de la Odontología. J Am Dent Assoc (ed. Esp) 2002;5:9-21.

Análisis completo, realizado por un comité de expertos, de la situación actual de la Odontología en casi todos sus aspectos y previsiones para el futuro inmediato. Aunque hace referencia a EUA, la mayoría de los aspectos son extrapolables a la realidad española.
12. Murdock SH, Hogue MN. Current patterns and future trends in the population of the United States: implications for dentistry and the dental profession in the twenty-first century. J Am Coll Dent 1998;65:29-35.

13. Jenicek M, Cleroux R. Epidemiología. Principios. Técnicas. Aplicaciones. Barcelona: Salvat, 1987.

14. American Dental Association. Future of dentistry. Chicago: American Dental Association, Health Policy Resources Center, 2001.

15**. Salsench J. Los retos de la odontología clínica. (Editorial). J Am Dent Assoc (ed. Esp) 2002;5:9.

Editorial claro, conciso y realista, que postula que el progreso técnico actual de la Odontología no puede ni debe eludir los principios básicos en los que se sustenta esta disciplina, cada vez más sofisticada.

16*. Nieto FM. EI Congreso de todos. (Editorial). RCOE 2003;8:13-4.

El autor expone la problemática y las dudas que se plantean a la hora de organizar un congreso ambicioso en el momento actual, plagado de ofertas formativas de todo tipo. 